\title{
Repetition effects in visual search
}

\author{
ANNE P. HILLSTROM \\ University of Wales, Bangor, Wales
}

\begin{abstract}
Maljkovic and Nakayama (1994) demonstrated an automatic benefit of repeating the defining feature of the target in search guided by salience. Thus, repetition influences target selection in search guided by bottom-up factors. Four experiments demonstrate this repetition effect in search guided by topdown factors, and so the repetition effect is not merely part of the mechanism for determining what display elements are salient. The effect is replicated in singleton search and in three situations requiring different degrees of top-down guidance: when the feature defining the target is less salient than the feature defining the response, when there is more than one singleton in the defining dimension, and when the target is defined by a conjunction of features. Repetition does not change the priorities of targets, relative to distractors: Display size affects search equally whether the target is repeated or changed. More than one mechanism may underlie the repetition effect in different experiments, but assuming that there is a unitary mechanism, a short-term episodic memory mechanism is proposed.
\end{abstract}

Visual search for a target amid multiple nontargets, or distractors, is a complex perceptual task that can be used to study visual attention. Most models of visual search have two stages involved in target selection (Egeth, 1977). The first is a preattentive stage that registers visual features in parallel and segments the visual scene into coarsely represented objects, according to principles such as those outlined by Marr (1982). This first stage also yields a prioritization of display elements that subsequently guides attention, a prioritization that is theorized to be influenced by both bottom-up factors (e.g., the perceptibility, or salience, of the object in the scene) and top-down factors (e.g., the foreknowledge and strategies of the searcher). The preattentive stage is followed by a stage in which attention is focused on elements according to the priorities assigned in the first stage, in order to identify the elements better and choose responses.

Memory is crucial to visual search: A representation of what defines the target must be held in memory in order to know when search has ended. Memory for target definitions is particularly important in the preattentive prioritization stage if the target is well defined, as when a searcher knows exactly what the target will look like. If the target is more conceptually defined-for instance, when the searcher knows only that the target will look different from the distractors--it is less obvious what sort of

This research was part of a doctoral dissertation submitted to the Johns Hopkins University under the direction of Steve Yantis. It was supported, in part, by a postdoctoral research fellowship, NRSA MH1 1064, from the National Institute of Mental Health, and by Grants RH-MH43924 from the National Institutes of Mental Health and SBR 9410406 from the National Science Foundation. I thank Steve Yantis, Bill Bacon, Shai Danziger, Brad Gibson, Peter Kramer, Gordon Logan, Cathleen Moore, and Kimron Shapiro for helpful discussions of this work. Correspondence concerning this article should be addressed to A. P. Hillstrom, Department of Psychology, Box 19528, University of Texas, Arlington, TX 76019-0528 (e-mail: a.hillstrom@uta.edu). explicit target template would be used to set priorities. But recent work by Maljkovic and Nakayama (1994) on repetition effects in search for salient targets has demonstrated a role for implicit memory in search that is guided largely by bottom-up factors, rather than by a well-elaborated target definition. The participants responded to the orientation of a target that was defined as a color singleton (either the single red element among green elements or the single green element among red elements). The participants identified the orientation of the color-singleton target more rapidly when the colors of the singleton and the distractors of the previous trial were repeated than when they were switched (i.e., when the current target color matched the preceding distractor color, and the current distractor color matched the preceding target color). Because the features defining the target were not the features defining the response (the defining and reported attributes, respectively, as they have been termed by Duncan, 1985), these repetition effects were attributed to selection of the target (prioritization) rather than selection of the response (see also Rabbitt, Cumming, \& Vyas, 1977, 1979). Because there was evidence of the repetition effect even when the searcher was given maximal topdown information about the target (i.e., when the participants were fully informed of the color of the singleton before each trial), the repetition effect was attributed to an automatic or implicit mechanism.

Maljkovic and Nakayama (1994) claimed that this implicit memory mechanism is the basis of pop-out of (noneffortful search for) salient targets. One could imagine this to be true in at least two ways. Memory for the defining feature could be used to facilitate the determination of what is salient. In other words, the repetition effect may be effectively making salient targets more salient. In this case, this mechanism should only affect search that is guided by bottom-up factors. Alternatively, the implicit memory could become an implicit top-down target tem- 
plate, in which case this mechanism could plausibly be functioning in all search, whether bottom-up or topdown. The purpose of this article is to demonstrate that a defining-feature repetition effect occurs in examples of search that are progressively less and less driven by target salience. If the repetition effect were specific to guidance by salience, it should have disappeared or become weaker when search was guided predominantly by topdown factors. But the repetition effect remained strong in these experiments, thus demonstrating that the repetition effect is a factor in both bottom-up and top-down guidance of search. The experiments that provide this demonstration also provide more information about the nature of the mechanism.

Although top-down and bottom-up guidance both can guide search, it is unclear whether they are selectable alternatives for guidance or whether bottom-up guidance is obligatory even when participants are using top-down guidance. It is clear that goals can override stimulus salience in guiding search (see, e.g., Folk, Remington, \& Johnston, 1992; Gibson \& Kelsey, 1998; Hillstrom \& Yantis, 1994; Yantis \& Egeth, 1999). And yet, there are effects of salient nontargets in search that suggest that bottom-up guidance may never be fully abandoned (Theeuwes, 1991). Because it is unknown whether bottomup guidance is obligatory, in the experiments reported herein, the involvement of bottom-up guidance is manipulated not by instructions to the searchers, but by the degree to which the targets are more salient than the distractors. If salience does contribute automatically to guidance in these experiments, it does not, by itself, allow the searcher to find the target.

The first step toward assessing whether the definingfeature repetition effect is confined to search by salience is to determine whether the repetition effect is impacted by the relative salience of the target-defining feature and the reported feature (Experiments 1 and 2 ). In previous research, the repetition effect was demonstrated only in search tasks in which the target-defining feature was more salient than the reported feature. By switching defining and reported features between Experiments 1 and 2, the salience of the defining feature is manipulated without changing the salience of the targets overall. Second, in Experiments 3 and 4 , two kinds of search are investigated in which there is an increasingly greater need for top-down guidance and bottom-up guidance becomes increasingly less effective. In Experiment 3, multiple singletons are in the display, thereby forcing the use of feature search (search for a target defined by a single, particular feature), rather than singleton search (search for a target defined as different from distractors in a particular dimension). In Experiment 4, the target was a conjunction of features that each occurs unconjoined in some of the distractors (conjunction search; e.g., search for a textured pink bar among solid pink and textured purple bars). Finally, Experiment 4 also probes what part of selection is affected by repetition: the time to prioritize elements or the level of priority assigned to elements.

In addition to defining-feature repetition effects, Maljkovic and Nakayama (1994) found effects of repeating the reported feature from trial to trial. This effect is reminiscent of the traditional repetition effects found in speeded reaction time (RT) tasks (e.g., Bertelson, 1961). In almost any of the visual search models, the response is chosen after the target is selected, in a final stage of processing. Therefore, the reported-feature repetition effect, because it affects response selection, would be based in the final stage of processing, according to such models. The defining-feature repetition effect, on the other hand, would be based earlier, in the prioritization process. Because the two types of repetition effects appear to be largely separable and the focus of this research is on how repetition affects target selection, the reported-feature repetition effect will be discussed only minimally herein.

\section{EXPERIMENT 1}

The first experiment, a fairly close replication of Maljkovic and Nakayama (1994), introduced the kinds of displays used in the remainder of the experiments reported in this article. The target was either a pink bar among purple bars or a purple bar among pink bars. Participants searched for a color singleton and reported its orientation. Thus, color was the defining feature, and orientation was the reported feature. A defining-feature repetition effect would be evidenced by more rapid responses to a target that was the same color as the preceding target (e.g., a pink singleton preceded by a pink singleton) than to a target that was a different color from the preceding target (a pink singleton preceded by a purple singleton). In order to distinguish the repetition effect from an expectation effect, search was conducted in alternating sequences, in which the participants knew exactly what color the target would be on each trial and the color changed every second trial, and in random sequences, in which the participants could not successfully anticipate what color the target would be on each trial. Search was also conducted in blocked sequences, in which the participants knew that the color of the target would change only once in the middle of the sequence (thereby maximizing the influence of repetitions in most of the trials).

\section{Method}

Participants. In all the experiments, undergraduate or graduate students at the Johns Hopkins University participated either to partially fulfill course requirements or for pay ( $\$ 5$ or $\$ 6$ ). Their ages ranged between 18 and 26 . All reported having normal or corrected-to-normal vision (except, as noted, for 1 participant in Experiment 4). All participated in only one experiment related to this research project. Twelve students $(6$ male) participated in Experiment 1.

Apparatus. A microcomputer controlled the presentation of stimuli and the collection of responses. Displays appeared on a 
Defining Feature (Color) Repeats

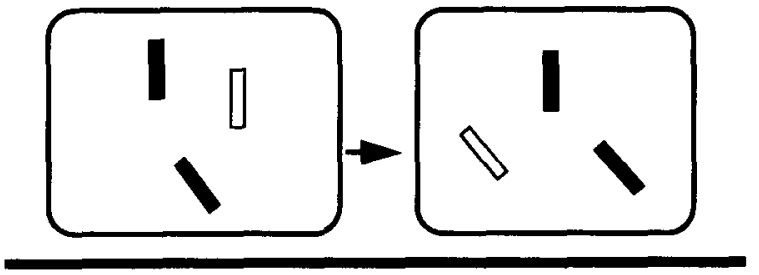

Reported Feature (Orientation) Repeats

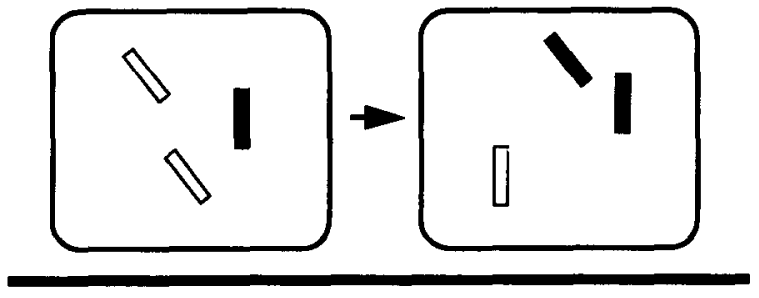

Both Features Repeat

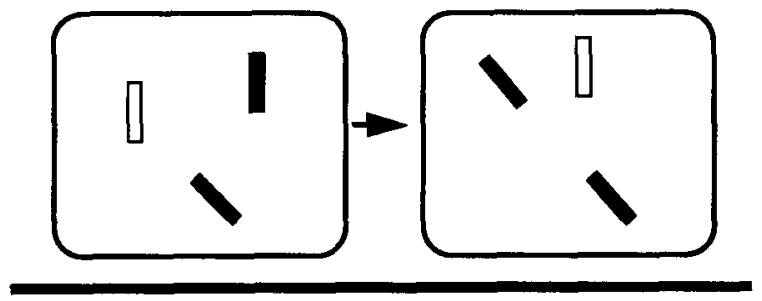

Neither Feature Repeats

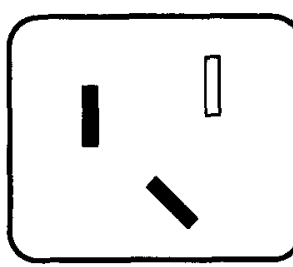

trial $n$

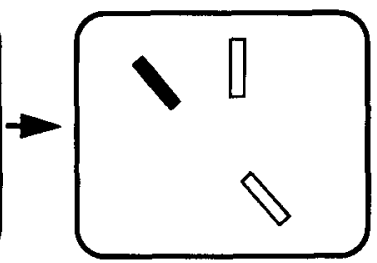

trial $n+1$

Figure 1. Schematic of the displays used in Experiment 1. The participants searched for a color singleton and reported its orientation.

Seiko Instruments CM-1450-C color monitor controlled by a VGA graphics card. Displays used $640 \times 350$ resolution graphics. Responses were collected with two of the buttons on a four-button response box. Testing was conducted with the participants seated alone and comfortably in a dimly lit, quiet room. This apparatus was used for all the experiments reported in this article.

Stimuli. In every search display, three rectangular bars were placed in randomly chosen positions in a circular array of five unmarked positions. The display background was black. The five positions in the circular array were evenly spaced around the circle, which had a radius of $1.9^{\circ}$ of visual angle, as measured from a typical viewing distance of $60 \mathrm{~cm}$. Each rectangular bar was $1.2^{\circ}$ long and $0.4^{\circ}$ wide.

The displays had the following characteristics. One bar, the target, was either pink or purple. The other two bars in the display were purple if the target was pink or pink if the target was purple. For the pink stimuli, activation levels were 25,0 , and 19 , for red, green, and blue guns, respectively, used to light up each pixel of the stimuli (on an activation scale of $0-63$ for each gun). For the purple stimuli, activation levels were 19,0 , and 25 , respectively, for the red, green, and blue guns. Each bar was randomly and independently set to be either vertically aligned or tilted $45^{\circ}$ to the left of upright. Figure 1 shows typical displays for the four kinds of transitions used in this experiment.

Procedure. At the start of the experiment, the participants were instructed about the task and told to respond as fast as possible while maintaining a high level of accuracy. The participants then were presented with a short practice block of 20 trials of search and then with nine regular blocks of 80 trials. At the end of each block, the accuracy rate and average RT for the block were presented during a rest period of $15-\mathrm{sec}$ duration. At the end of the fifth block, a longer break was given. The participants left the testing room for at least 2 min during that break. The total duration of a testing session was shorter than $\mathbf{4 5} \mathrm{min}$.

The nine blocks of trials were divided equally among the blocked, alternating, and random sequences of target color, and the blocks were presented in random order, preceded by one practice block randomly assigned to one of the three sequences. In all the blocks, there were 40 pink targets and 40 purple targets. In the blocked sequence, all the trials with pink targets were presented, then all the trials with purple targets. In this block, the participants had foreknowledge of what target would appear on each trial, and almost all the trials in the block were repetition trials. In the alternating sequences, two trials with pink targets were followed by two trials with purple targets, followed by two pink targets, followed by two purple targets, and so forth throughout the 80 trials. In this block, the participants had foreknowledge of what target would appear on each trial, and the trials were equally divided between repetition and alternation trials. In the random sequence, the order of the pink and the purple targets was pseudorandom within the block. In this block, which had approximately equal numbers of repetition and alternation trials, the participants had no foreknowledge of what target would appear on each trial.

Before each block, a message on the monitor informed the participant what sequence would occur in the block and suggested using that information to improve response efficiency, if possible. There were no practice trials at the beginning of each block.

Each trial consisted of a search display, presented until either the participant responded or $2,500 \mathrm{msec}$ had elapsed, followed by an 800 -msec response-stimulus interval (RSI), during which the screen was black. ${ }^{\text {T }}$ The participants responded by pressing the left button with the left index finger if the target (i.e., the color singleton) was tilted or the right button with the right index finger if the target was vertical. If the wrong response was given to a search display, a $200-\mathrm{Hz}$ tone sounded for $200 \mathrm{msec}$ during the RSI, to inform the participant of the error. The computer recorded RT to the nearest millisecond.

\section{Results}

In this and all subsequent experiments, data from the practice block and the first block of each sequence type were not analyzed. When considering the effect of repetition, each trial played two roles: It both influenced subsequent trials and was influenced by preceding trials. When analyzing RT, trials on which errors were made were analyzed neither as an influenced nor as an influencing trial: Neither the pair beginning with the error trial nor the trial pair ending with the error trial was analyzed. Trials with particularly rapid responses ( $<200 \mathrm{msec}$ ) or with no responses before the response deadline $(2,500 \mathrm{msec}$ in this experiment) were considered error trials. In this experiment, fewer than $0.5 \%$ of the trials for any participant were discarded because of extreme RTs.

First-order repetition effects. Panel A of Figure 2 shows the mean RTs when target color was repeated or 

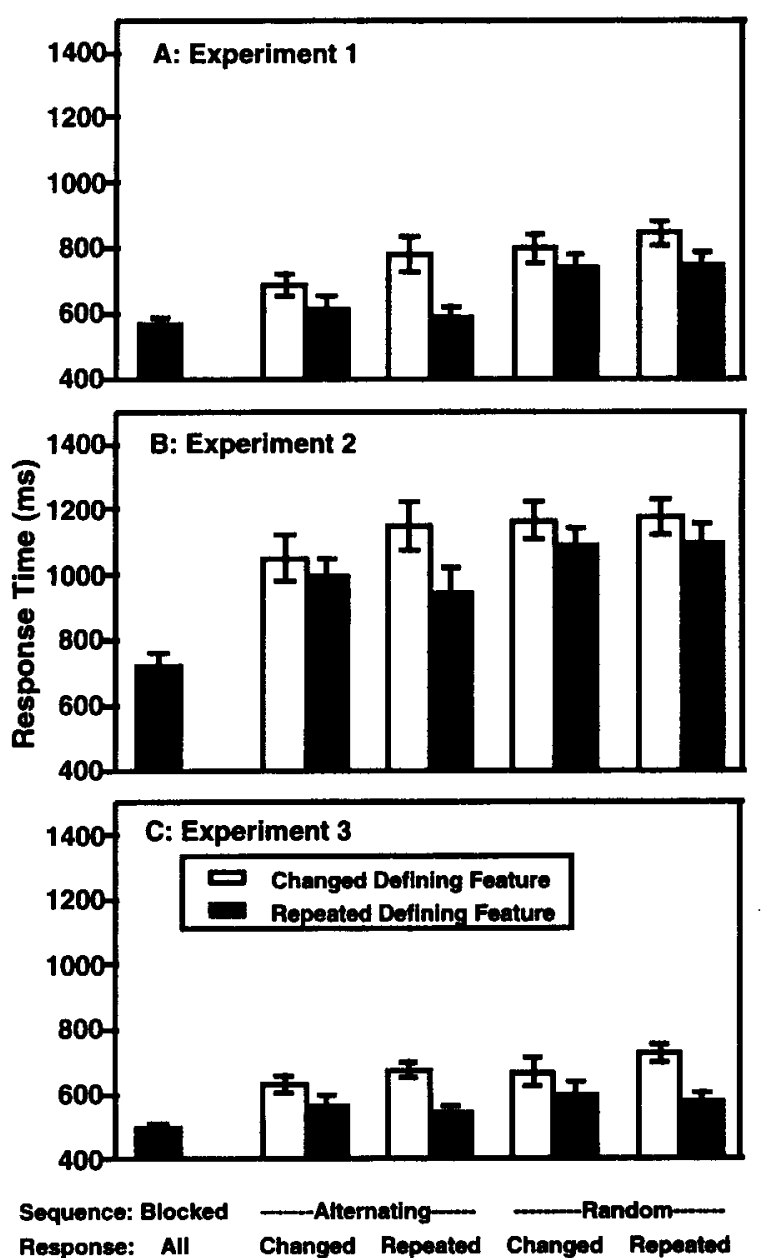

Figure 2. Response times (RTs) for Experiments 1-3. In Experiment 1 , the participants searched for a color singleton and reported its orientation. In Experiment 2, the participants searched for an orientation singleton and reported its color. In Experiment 3 , the participants searched for a target with a precued color and reported its orientation. $R T$ is a function of trial sequence, defining-feature repetition, and reported-feature repetition (labeled response in the graph). Error bars represent betweensubjects standard error in this and all subsequent graphs.

was changed in the alternating, random, and blocked trial sequences. RTs for trials in the alternating and random sequences were analyzed in a repeated measures analysis of variance (ANOVA) with three factors: trial sequence (target color alternating between trials or random), defining-feature repetition (target color the same as that in the preceding trial or different from that in the preceding trial), and reported-feature repetition (target orientation same as or different from that in the preceding trial). An alpha level of .05 was used for all statistical tests in this article, and any main effects or interactions that are not discussed were not significant.

Repeating the defining feature of the target speeded responses by an average of $105 \mathrm{msec}[F(1,11)=28.5]$. Repeating the reported feature slowed search by an aver- age of $31 \mathrm{msec}[F(1,11)=10.6]$. There was an interaction between defining-feature repetition and reported-feature repetition $[F(1,11)=11.4]$. Repeating the defining feature speeded search both when the response feature was repeated $(146 \mathrm{msec})$ and when it was changed [ $64 \mathrm{msec}$; $F(1,11)=26.0$ and $F(1,11)=16.5$, respectively]. On the basis of panel A of Figure 2, it appears that the effect of repeating the reported feature depended on whether the defining feature was repeated. When the defining feature was changed, responses were faster if the reported feature also was changed; if the defining feature was repeated, repetition of the reported feature either speeded responses or had little impact.

Responses were $115 \mathrm{msec}$ faster, on average, to trials in the alternating sequences than to trials in the random sequences $[F(1,11)=15.7]$, a result that reflects expectancy. Sequence also influenced the size of the defining-feature repetition effect $[F(1,11)=8.2]$ : The defining-feature repetition effect was larger for the alternating sequence $(132 \mathrm{msec})$ than for the random sequence $(78 \mathrm{msec})$, but simple effects analyses showed that it was significant for both $[F(1,11)=27.0$ and $F(1,11)=24.1$, respectively].

First-order effects on accuracy. The participants made incorrect responses on an average of $2.1 \%$ of all trials (ranging from $0.4 \%$ to $5 \%$ across participants). Table 1 shows the error rates for the alternating and random sequence conditions. The error rates were analyzed by means of an ANOVA identical to that used to analyze RTs.

The influence of defining-feature repetition was not significant $[F(1,11)=1.7]$. Thus, the defining-feature repetition effect was not due to a simple speed-accuracy tradeoff: The participants were not more accurate in the condition in which they were slower (changed definingfeature condition). Significantly, more errors were made when the reported feature was changed than when it was repeated $[F(1,11)=6.8]$, and there was a significant interaction between reported-feature repetition and definingfeature repetition $[F(1,11)=7.8]$. When the defining feature was changed, reported-feature repetition did not influence errors rates; when the defining feature was repeated, repeating the reported feature resulted in fewer errors. It appears that defining-feature repetition had limited influence on accuracy in this experiment.

Higher order repetition effects. Panel A of Figure 3 presents RTs in the random sequences as a function of the match or mismatch of the defining feature on the current trial (trial $n$ ) to the defining feature used $i$ trials ago (trial $n-i) .^{2}$ For example, the two data points presented for trial -2 show the RT for trial $n$ when the defining feature for trial $n$ is either the same as or different from the defining feature for trial $n-2$. This way of looking at the data disregards the effect of intervening trials. The graph suggests that the defining features on trials $n-1$ through $n-3$ all influence RTs. One-tailed $t$ tests were done, comparing RTs when the defining feature for trial $n-i$ matched the defining feature for trial $n$ with RTs when the defining feature for trial $n-i$ did not match the defining feature for trial $n$ (one-tailed, rather than 
Table 1

Error Rates (\%) in Experiments 1-3

\begin{tabular}{|c|c|c|c|c|c|}
\hline \multirow[b]{2}{*}{ Experiment } & \multirow[b]{2}{*}{ Trial Sequence } & \multicolumn{2}{|c|}{ Reported Feature Changed } & \multicolumn{2}{|c|}{ Reported Feature Repeated } \\
\hline & & DF Changed & DF Repeated & DF Changed & DF Repeated \\
\hline \multirow[t]{2}{*}{1} & Alternating & 0.9 & 2.7 & 1.1 & 0.4 \\
\hline & Random & 1.0 & 1.8 & 0.7 & 0.7 \\
\hline \multirow[t]{2}{*}{2} & Alternating & 3.1 & 7.9 & 4.6 & 4.3 \\
\hline & Random & 4.1 & 3.6 & 5.8 & 5.0 \\
\hline \multirow[t]{2}{*}{3} & Alternating & 2.8 & 3.3 & 7.4 & 1.5 \\
\hline & Random & 2.8 & 2.5 & 5.7 & 1.2 \\
\hline
\end{tabular}

Note-DF, defining feature.

two-tailed, tests were done because there were clear expectations of the direction of the effect being sought). The $t$ tests showed that the difference in RTs were significant for influencing trials $n-1$ and $n-3[t(11)=5.1$ and $t(11)=2.1$, respectively], but not for trials $n-2$ and $n-4$ through $n-7[t(11)=1.6,1.0,0.8,0.7$, and 0.5 , respectively]. The same comparisons of the "influence" of trial $n+1$ through trial $n+7$ on trial $n$ are included to give a rough suggestion of how much variance could be attributed to something other than memory. Clearly, future trials should not (and did not) influence RTs.

Panel B of Figure 3 shows the influence of same or different reported feature in preceding or subsequent trials. As was demonstrated in the analyses of first-order effects, reported-feature repetition tended to slow responses, not speed them. One-tailed $t$ tests like those reported in the preceding paragraph showed that the reported feature of trial $n-1$ influenced RT [ $t(11)=-1.9]$ but that no preceding reported feature influenced RT $[t(11)=0.2,0.4$, $0.0,0.9,-0.9$, and -0.5 for trials $n-2$ through $n-7$, respectively].
In order to determine the nature of the higher order effects, two more tests were done. To determine whether the effect was cumulative, the influence of the number of consecutive repetitions on the size of the effect was measured (see Figure 4). There was an insufficient number of trials to analyze the effect of more than three repetitions. A one-way repeated measures ANOVA of the influence of one to three repetitions confirmed that there was a significant effect of the number of consecutive definingfeature repetitions $[F(2,22)=4.7]$. A longer string of defining-feature repetitions resulted in a faster RT. A separate one-way repeated measures ANOVA demonstrated that the number of consecutive reported-feature repetitions (one, two, or three) did not significantly influence RT $[F(2,22)<1]$.

To determine whether the repetition effect of a single trial decays, a measure was taken of the effect of the number of trials since the defining feature of the target was the same as the current defining feature (see Figure 5). For example, if the current target is pink and the previous two targets were purple, the last time the defining feature
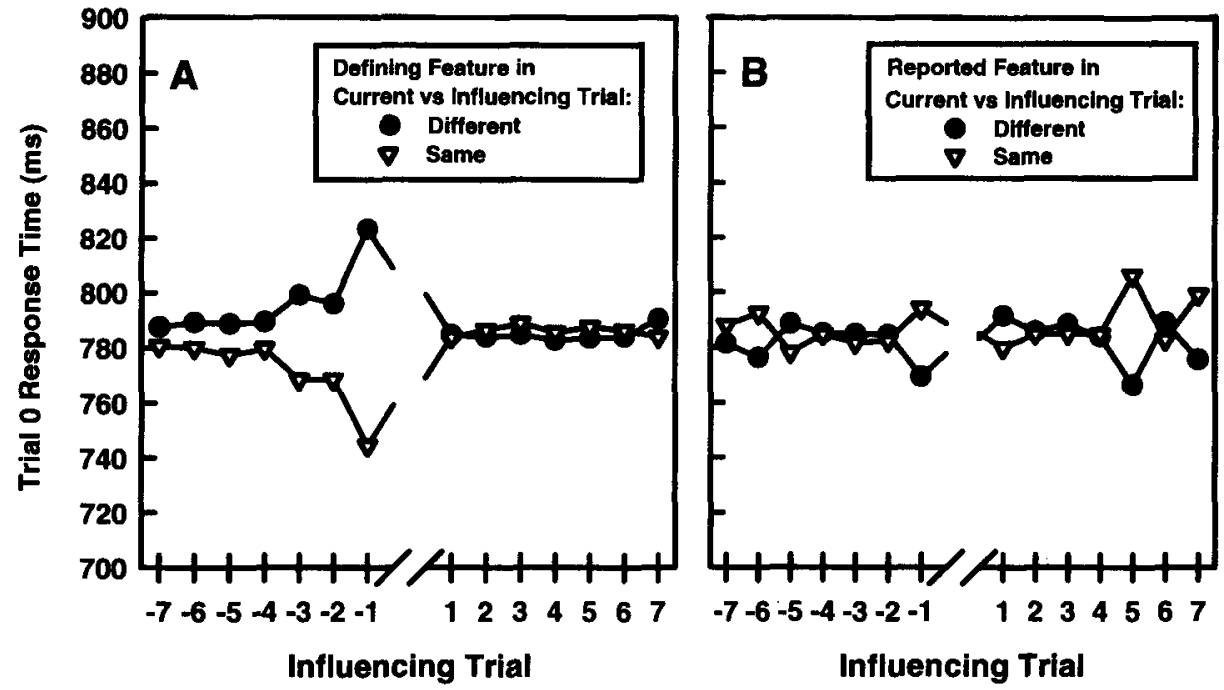

Figure 3. (A) Experiment 1 response times, as a function of the match between the current defining feature and the defining feature in a preceding (or subsequent) trial. (B) Experiment 1 response times, as a function of the match between the current reported feature and the reported feature in a preceding (or subsequent) trial. 


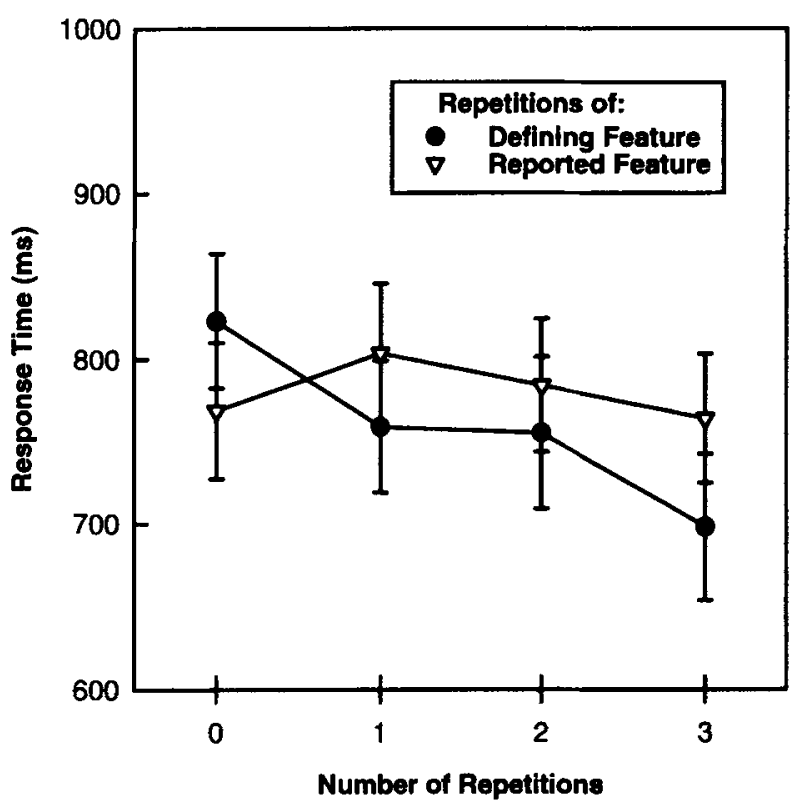

Figure 4, Experiment 1 response times, as a function of the number of consecutive repetitions of the defining or the reported feature.

was the same as that in the current trial was three trials ago. The few trials that followed more than three consecutive alternate-color trials were discarded from this analysis. A one-way repeated measures ANOVA confirmed that there was a significant effect of the number of trials since the last occurrence of a matching defining feature $[F(3,33)=7.0]$. RTs were more rapid when fewer alternatecolor trials intervened. Although Figure 5 suggests that RTs were more rapid when more alternate-orientation trials intervened, a one-way repeated measures ANOVA did not yield a significant effect of the number of trials since the last occurrence of a matching reported feature $[F(3,33)=2.8]$.

\section{Discussion}

The results of Experiment 1 replicated Maljkovic and Nakayama's (1994) results for the most part. Repetition of the defining feature and repetition of the reported feature both influenced search. There was an effect of repetition beyond that provided by foreknowledge of what target to expect. The effect was cumulative when the target was repeated more than once, and the effect decayed gradually when repetitions ceased. Thus, any mechanism that accounts for repetition effects in models of visual search must allow for the possibility of lingering, cumulative influences.

Experiment 1 revealed the existence of higher order defining-feature repetition effects. The defining-feature repetition effect became larger when the number of consecutive repetitions increased and became smaller as the number of intervening trials since the last matching defin- ing feature grew. Thus, the effect was cumulative and decayed over intervening trials (or time).

The effect of using a defining feature was less resistant to decay or interference by intervening trials in the present experiment (three preceding trials influence the current trial) than in Maljkovic and Nakayama's (1994) experiments (five to seven preceding trials influenced the current one). It is not clear why this difference obtained, but a number of differences between the experiments may have contributed to this, including the fact that the present data were collected from participants less practiced than Maljkovic and Nakayama's participants. It is possible that this difference in experience with the task might have led to strategic differences in how the task was approached. If this speculation is true, the strategy affected only higher order effects, not the effect of the most recent trial.

The present paper addresses the effect of repeating the defining feature independent of repetition effects for the reported feature. As such, the statistical interaction between the first-order defining-feature repetition effect and the first-order reported-feature repetition effect could be seen to challenge the independence of the two effects. However, when participants are asked to respond to a single dimension of multidimensional stimuli, it is common to find that repetition of the reported dimension interacts with repetition of the irrelevant dimension (Fletcher, 1981; Hommel, 1998; Lockhead, Gruenewald, \& King, 1978). Such an interaction typically takes the following form. When the reported feature is changed, responses are sometimes faster if the irrelevant feature also is changed.

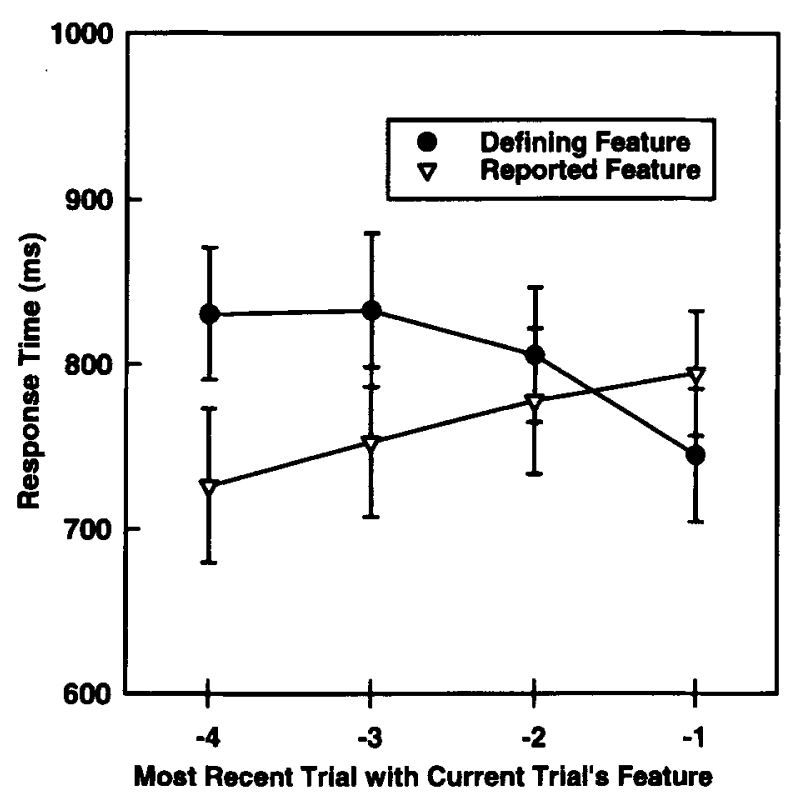

Figure 5. Experiment 1 response times, as a function of the recency of the most recent trial with the same defining or reported feature. 
When the reported feature is repeated, responses are faster if the irrelevant feature also is repeated. So, complete repetition of the stimulus is best, and complete change of the stimulus is sometimes better than partial change of the stimulus. The results of Experiment 1 show a similar pattern, except that repetition of the defining feature is always associated with faster responses than is change to the defining feature. Thus, in this experiment, responses are faster when the defining feature is repeated and the reported feature is changed than when the defining feature is changed and the reported feature is repeated. This is inconsistent with what was found by the earlier reportedfeature repetition effect experiments, in which responses were faster when the reported feature was repeated and the irrelevant feature was changed than when the irrelevant feature was repeated and the reported feature was changed. In the experiments reported here, the defining feature might have a dual role, as follows. The defining feature drives the defining-feature repetition effect in prioritization. Once a target has been attended, it is a multidimensional stimulus, where the reported feature is relevant to the response and the defining feature is irrelevant. At this point, the defining feature of the target interacts with the reported feature to affect the reported-feature repetition effect.

\section{EXPERIMENT 2}

In Experiment 1, as in all of Maljkovic and Nakayama's (1994) experiments, the defining feature (color or spatial frequency) seemed to be more salient than the reported feature (orientation or direction of shift of vernier acuity bars, respectively), although salience was not measured. Experiment 2, by reversing the roles of the features used in Experiment 1, tested whether the role (defining vs. reported) or the nature (color vs. orientation, more vs. less salient) of the feature determines the repetition effect. Virtually the same displays were used in Experiment 2 as in Experiment 1: The orientations and colors were the same; only their roles as defining or reported feature were changed.

Although the salience of color and orientation were not measured directly, there is every reason to believe that the variation in orientation in Experiments 1 and 2 was less salient than the variation in color. Orientation singletons are in general less salient than color singletons; to equate singleton pop-out in the two dimensions, color variation must be made quite small and orientation variation quite large (see, e.g., Theeuwes, 1991). In Experiment 1, no attempt was made to equate the salience of the color and orientation variations, and the subjective impression was that the color differences were much more salient than the orientation differences. Therefore, the defining feature in Experiment 2 (orientation) should have been less salient than the defining feature in Experiment 1 (color).

\section{Method}

Experiment 2 was the same as Experiment 1, except as follows.
Participants. Twelve students (4 female) participated in the experiment.

Stimuli. One bar, the target, was either vertical or tilted $45^{\circ}$ to the left of upright. The other two bars in the display were tilted $45^{\circ}$ if the target was vertical or were vertical if the target was tilted. Each bar was randomly and independently chosen to be either pink or purple.

Procedure. There were 10 practice trials at the start of the experiment. The trial sequences were as follows. In the blocked sequence, the participants saw 40 vertical targets, followed by 40 tilted targets. In the alternating sequence, the sequence of target orientations was vertical-vertical-tilted-tilted-vertical-vertical-tilted-tilted-, and so forth. In the random sequence, there were 40 trials with vertical targets and 40 with tilted targets, and the order was random. The participants pressed the left button with the left index finger if the target (i.e., the orientation singleton) was pink or the right button with the right index finger if the target was purple.

\section{Results}

No trials were discarded because of rapid responses $(<200 \mathrm{msec})$. No more than $1 \%$ of the trials for any participant were discarded because of slow responses $(>2,500$-msec deadline). The same analyses were performed for this experiment as for Experiment 1. RTs were slower (Figure 2, panel B), and error rates were higher (Table 1) in this experiment than in Experiment 1, demonstrating that, as was expected, orientation singletons were less salient than color singletons. ${ }^{3}$

First-order repetition effects. Panel B of Figure 2 shows the mean RTs when target orientation was repeated or was changed in the alternating, random, and blocked trial sequences. Repeating the defining feature (orientation) speeded responses by a mean of $105 \mathrm{msec}[F(1,11)=22.5]$, which is equivalent to the magnitude of the repetition effect in Experiment 1. The 17-msec slowing of RT when the reported feature (color) was repeated was nonsignificant $[F(1,11)=1.1]$, but there was an interaction between defining-feature repetition and reported-feature repetition $[F(1,11)=5.6]$. Repeating the defining feature speeded responses both when the reported-feature repeated (144 msec) and when it was changed [67 msec; $F(1,11)=21.5$ and $F(1,11)=8.2$, respectively]. Panel B of Figure 2 shows that the interaction was like that observed in Experiment 1: Repeating the reported feature appears to have speeded responses only when the defining feature was repeated and the participants knew in advance that it would be (alternating sequence, repeated defining feature). In the other conditions, repeating the reported feature tended to slow responses. Presenting target orientations in an alternating rather than a random sequence speeded RTs significantly [ $97 \mathrm{msec}$ faster, on average; $F(1,11)=5.6$ ]

First-order effects on accuracy. Error rates averaged $4.1 \%$ (ranging from $1.5 \%$ to $7.7 \%$ across participants). The error rates for the alternating and random sequences are shown in Table 1 . The defining-feature repetition effect cannot be attributed to a simple speed-accuracy tradeoff. Not only did the participants respond faster when the defining feature was repeated than when it was changed, they made significantly fewer errors $[F(1,11)=8.1]$.

Higher order repetition effects. Panel A of Figure 6 shows the influence of trial $n-i$ on trial $n$, where $i$ ranged 

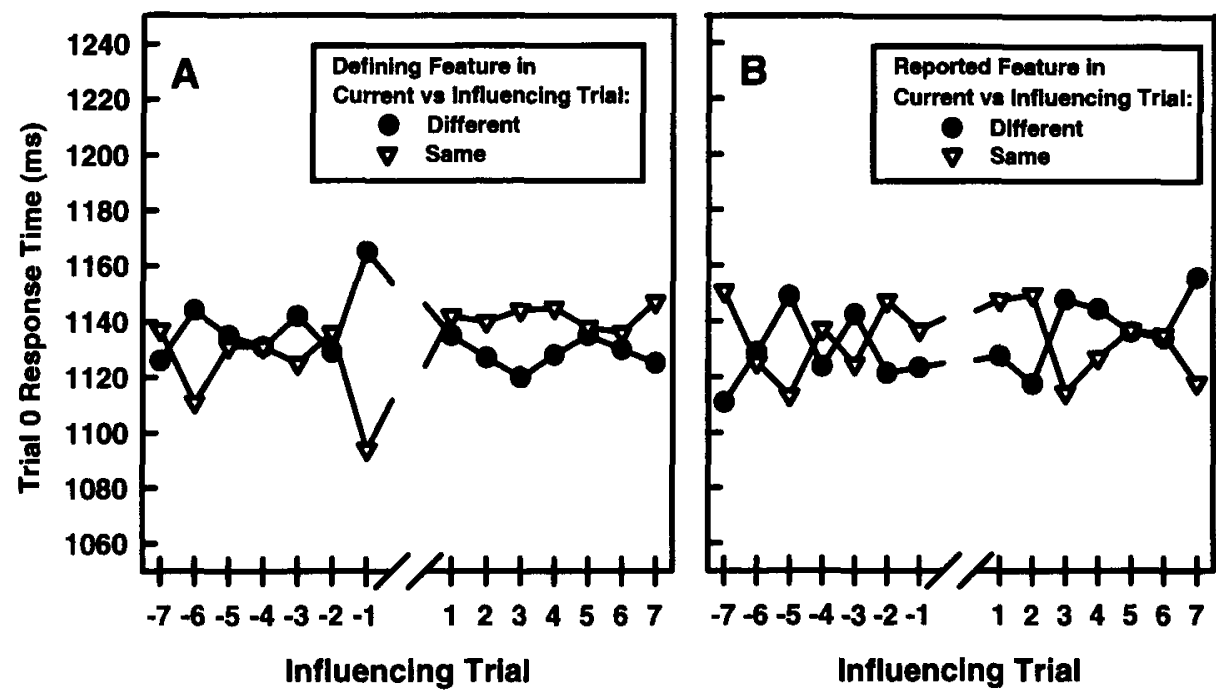

Figure 6. (A) Experiment 2 response times, as a function of the match between the current defining feature and the defining feature in a preceding (or subsequent) trial. (B) Experiment 2 response times, as a function of the match between the current reported feature and the reported feature in a preceding (or subsequent) trial.

from 7 to -7 . One-tailed Student's $t$ tests confirm what panel A shows, that match or mismatch of the most recent defining orientation significantly influenced RTs on the current trial $[t(11)=2.6]$ and that no other trial except trial -6 had a significant influence $[t(11)=-0.4$, $1.1,0.0,0.2,2.4$, and -0.6 for $i$ from 2 to 7 , respectively]. Presumably, the influence of trial -6 is coincidental (a Type I error). Panel B of Figure 6 shows the influence of the reported feature (color) of preceding and subsequent trials on the current trial. One-tailed Student's $t$ tests showed that the reported feature of preceding trials did not significantly influence RTs $[t(11)=-0.6,-1.3,0.8$, $-0.9,1.4,0.1$, and -1.7 , for trials $n-1$ through $n-7$, respectively].

Figure 7 shows RT as a function of the number of consecutive repetitions of the defining or the reported feature, and Figure 8 shows RT as a function of how recently the same defining feature or reported feature was seen. There was no evidence that the number of consecutive repetitions of the defining feature influenced RT significantly $[F(2,22)<1]$. In other words, RTs for the first, second, or third consecutive repetition of a defining feature could not be reliably distinguished. There was also no evidence that the number of repetitions of the reported feature influenced RT $[F(2,22)=1.2]$. Although Figure 8 shows a trend for immediately repeated defining features to be responded to faster than trials with less recent matches to the defining feature, recency of a matching defining feature had no significant influence on response time $[F(3,33)=$ 1.1]. There was also no significant effect of the recency of a matching reported feature $[F(3,33)=2.0]$. Together, these analyses of higher order repetition effects show that only the most recent defining feature influences responses to the current target and that no preceding reported features influence responses.

\section{Discussion}

In Experiment 2, as in Experiment 1, repeating the defining feature speeded search and repeating the reported feature tended to slow search slightly. It is the role of the feature (defining or reported), not the nature of the feature (color or orientation, more or less salient), that determines the repetition effect.

The effect of the most recent trial was the same in Experiment 2 as in Experiment 1. However, no higher order repetition effects were observed in Experiment 2. In Experiment 1 , the repetition effect was cumulative over consecutive repetitions and decayed slowly after a switch. In Experiment 2, multiple consecutive repetitions had no stronger effect than a single repetition, and the effect of using a particular defining feature did not influence any trial but the one immediately following it.

This lack of a higher order effect may have been due to the slower responses to trials; the residual effect may have decayed before two trials were presented. However, the longer time between trials by itself could not explain the lack of effect. The higher order effect in Experiment 1 lasted for at least three trials, which took more time than two trials in Experiment 2. Decay would have had to be more rapid in Experiment 2, perhaps because the targets were more similar to the distractors. Such a result is reminiscent of the phenomenon in short-term forgetting that memory for stimuli decays faster when the stimuli to be remembered are similar to one another (see, e.g., Posner \& Konick, 1966; Wickelgren, 1965).

Alternatively, there could be two bases for repetition effects-one for bottom-up search and the other for topdown search. The orientation singletons used in Experiment 2 were less salient than the color singletons used in Experiment 1. For this reason, the participants might have used more top-down guidance in Experiment 2. In the ran- 


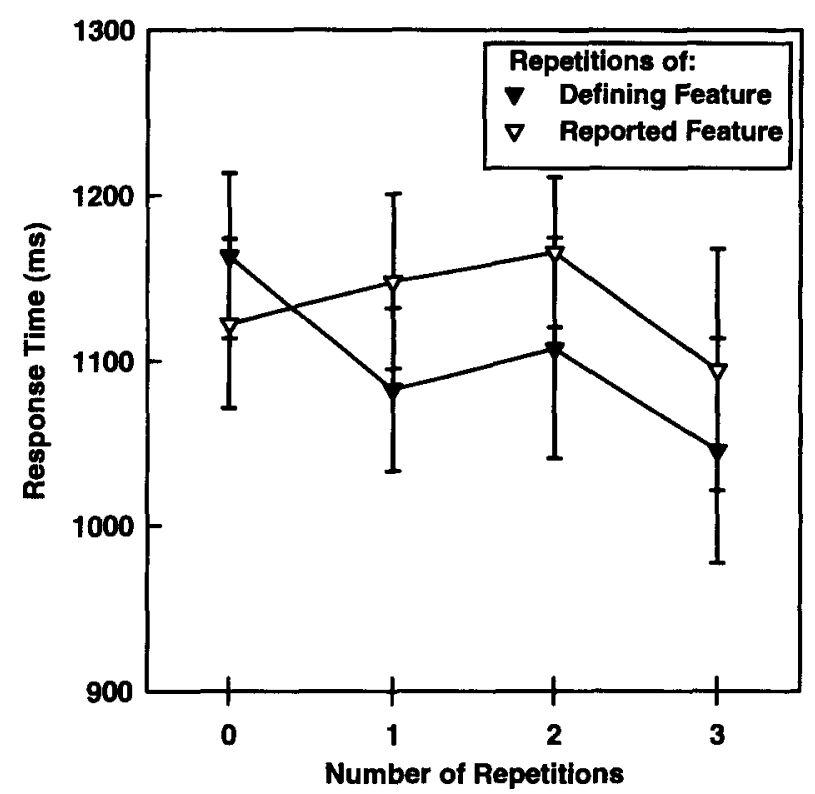

Figure 7. Experiment 2 response times, as a function of the number of consecutive repetitions of the defining or the reported feature.

dom sequence blocks (the blocks in which higher order effects were sought), the participants would have had no foreknowledge about the target-defining feature, so topdown strategies would be, at best, nonoptimal. But if the lessened salience of the Experiment 2 targets forced more reliance on strategic search, repetition effects could have affected whether the search strategy chosen favored one target over the other.

\section{EXPERIMENT 3}

Although the differences in higher order effects in the first two experiments suggest that repetition effects may work differently for top-down and bottom-up search, it is by no means clear that there were top-down influences on search in those experiments. The target sequence manipulation was used to encourage the participants to change their reliance on top-down versus bottom-up guidance between blocks, but targets could have been found by using only bottom-up guidance in all the blocks of both experiments. Bacon and Egeth (1994) have shown that participants often adopt a singleton-search strategy, or a strategy of searching for local feature mismatches, when they are not obliged to detect the presence of a specific defining value to find the target (see also Duncan \& Humphreys, 1989).

In order to determine whether repetition effects are observed in top-down search, in Experiment 3, displays required the participants to use top-down guidance to find the targets. The participants searched for targets defined by color. There were two color singletons (and two distractors sharing a color) in each display. An auditory tone before each trial indicated which color defined the target. The participants reported the orientation of the target. The presence of more than one color singleton in each display forced the participants to adopt a top-down search strategy (Bacon \& Egeth, 1994). If the defining-feature repetition effect obtains only in bottom-up search, there would be no defining-feature repetition effect in this experiment. If it obtains in top-down search, the effect in this experiment should be as strong as, if not stronger than, the effect found in Experiments 1 and 2.

\section{Method}

Participants. Twelve students ( 3 male) participated in the experiment.

Stimuli. In every search display, four rectangular bars were placed in randomly chosen positions in a circular array of six unmarked positions against a black background. The positions in the circular array were all centered $1.9^{\circ}$ of visual angle from the center of the circle. There was always one red, one blue, and two green bars in each display. Two bars, chosen randomly, were vertical, and the other two were tilted $45^{\circ}$ to the left of upright. The auditory tones used to indicate which color defined the target lasted for $100 \mathrm{msec}$. The tone was $1600 \mathrm{~Hz}$ if the target was red or $800 \mathrm{~Hz}$ if the target was blue.

Procedure. The procedure was the same as that in Experiment 1, except for the following. The practice block at the start of the experiment consisted of 20 trials of search. Each trial began $800 \mathrm{msec}$ after the previous response. The first thing presented was an auditory tone that indicated the color of the target for the trial. Although blocked, alternating, and random sequences of target colors were used, as in Experiment 1, the cue preceding each trial in this experiment enabled the participants to know exactly what target to expect in all three sequences. The search display appeared $100 \mathrm{msec}$ after the cue tone ended. Wrong responses were signaled by a double beep $(200 \mathrm{~Hz})$.

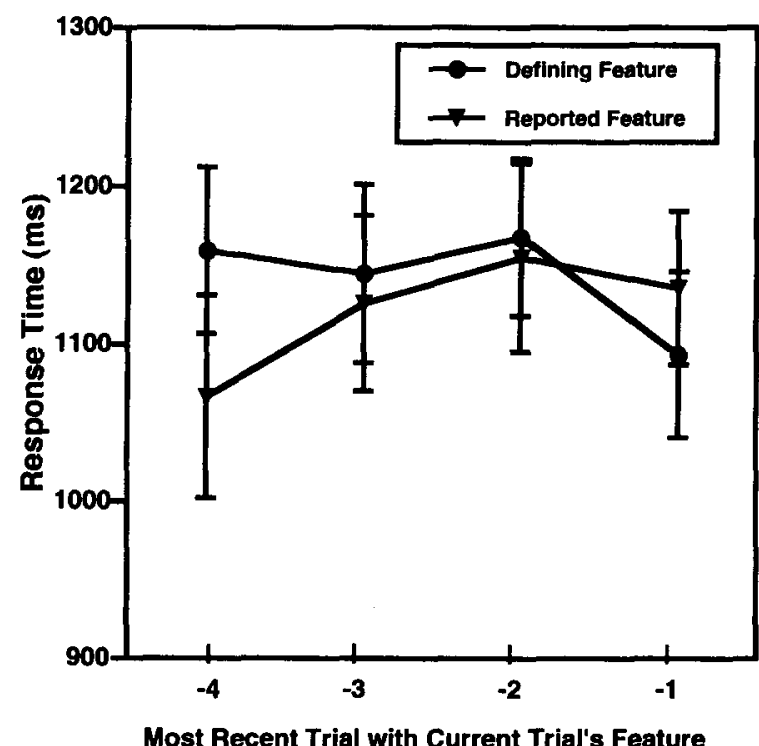

Figure 8. Experiment 2 response times, as a function of the recency of the most recent trial with the same defining or reported feature. 

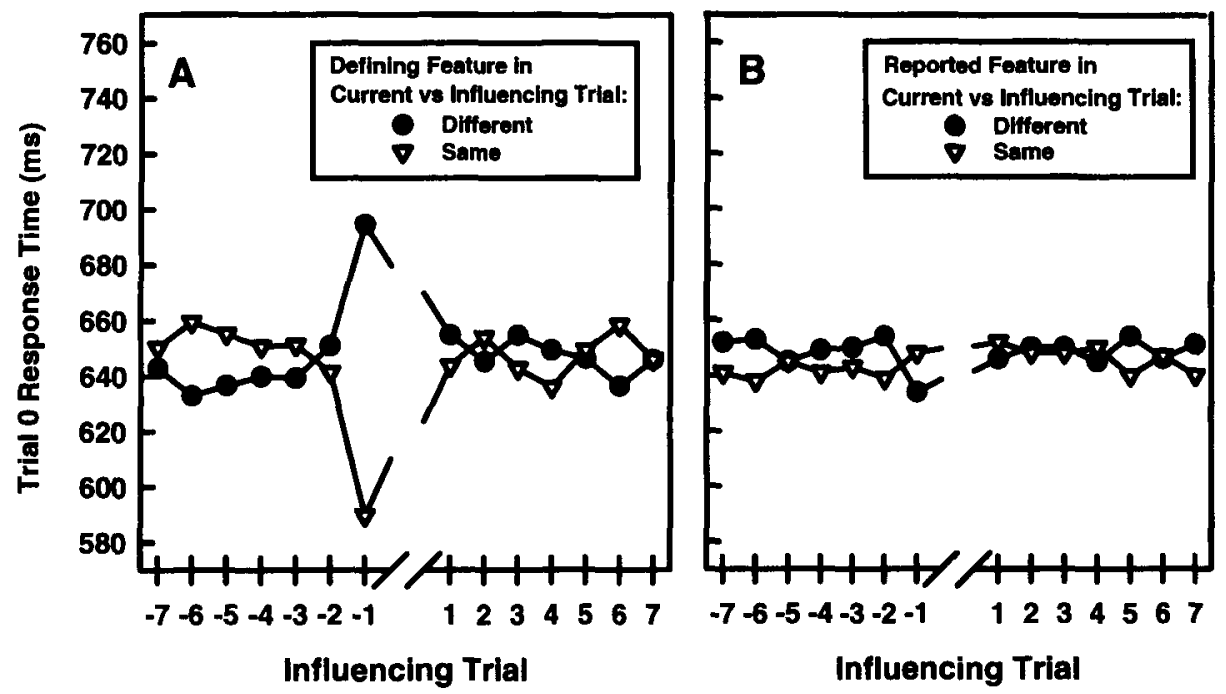

Figure 9. (A) Experiment 3 response times as a function of the match between the current defining feature and the defining feature in a preceding (or subsequent) trial. (B) Experiment 3 response times as a function of the match between the current reported feature and the reported feature in a preceding (or subsequent) trial.

\section{Results}

Fewer than $0.5 \%$ of the trials for any participant were discarded because of particularly fast or slow responses. Overall, responses were faster in Experiment 3 than in Experiments 1 and 2 . This speed-up most likely reflects, in part, the more salient colors used in Experiment 3, and it probably also reflects the use of the top-down information given in advance of the trial about the defining feature of the target. The same analyses were performed for this experiment as for Experiments 1 and 2.

First-order repetition effects. Panel C of Figure 2 shows RT as a function of the trial sequence and of defining-feature and reported-feature transitions. Because the participants were required to use top-down information to find the target in both the alternating and the random sequences, the sequences did not differ substantially in terms of target expectancy, as they did in Experiments 1 and 2 . In support of this, the effect of trial sequence did not reach significance $[F(1,11)=3]$.

Repeating the defining feature speeded responses by $104 \mathrm{msec}[F(1,11)=43.4]$. Repeating the reported feature significantly slowed RT $[F(1,11)=8.8]$, and there was an interaction between reported-feature repetition and defining-feature repetition $[F(1,11)=20.2]$. Repeating the defining feature speeded RT both when the reported feature was repeated $(138 \mathrm{msec})$ and when it was changed $[72 \mathrm{msec} ; F(1,11)=39.6$ and $F(1,11)=38.1$, respectively]. When the defining feature was repeated, repeating the reported feature speeded RTs; when the defining feature was changed, repeating the reported feature slowed RTs.

First-order effects on accuracy. Error rates averaged $3.1 \%$ (ranging from $1.0 \%$ to $4.4 \%$ across participants). Error rates are shown in Table 1 . There were significantly fewer errors made when the defining feature of the target was repeated than when it was changed $[F(1,11)=$ 13.8]. Because the participants responded both more accurately and faster when the defining feature was repeated, the defining-feature repetition effect cannot be attributed to a simple speed-accuracy tradeoff. Reported-feature repetition did not significantly affect error rate $[F(1,11)=$ $2.2]$, but the interaction between reported-feature repetition and defining-feature repetition did $[F(1,11)=13.3]$. The interaction appears to show an advantage for accuracy when both the defining and the reported feature were repeated and a disadvantage when the defining feature was changed while the reported feature was repeated.

Higher order repetition effects. Panel A of Figure 9 presents RT as a function of the match or mismatch of the defining feature, on the current trial to the defining feature used in a preceding or subsequent trial. One-tailed Student's $t$ tests showed that when the defining feature on trial $n-1$ was the same as the current defining feature, RT was significantly faster $[t(11)=4.8]$. No other trial preceding the current one had a positive repetition effect. Trials $n-3$ through $n-7$ appear to tend toward an inhibitory effect. The effect was significant for trial $n-6$ $[t(11)=-2.5]$, but not for the other trials $[t(11)=1.1$, $-1.0,-1.6,-1.7$, and -0.8 for trials $n-2, n-3, n-$ $4, n-5$, and $n-7$, respectively]. Panel B of Figure 9 shows the influence of matching or mismatching reported features in trials $n-7$ to $n+7$. Student $t$ s for trials $n-$ 1 through $n-7$ were $-1.4,2.0,0.7,0.7,0.0,1.2$, and 1.1 , respectively. The advantage for a repeated reported feature from trial $n-2$ was significant, by a one-tailed criterion.

Figure 10 shows that RT was significantly influenced by the number of consecutive defining-feature repetitions, 


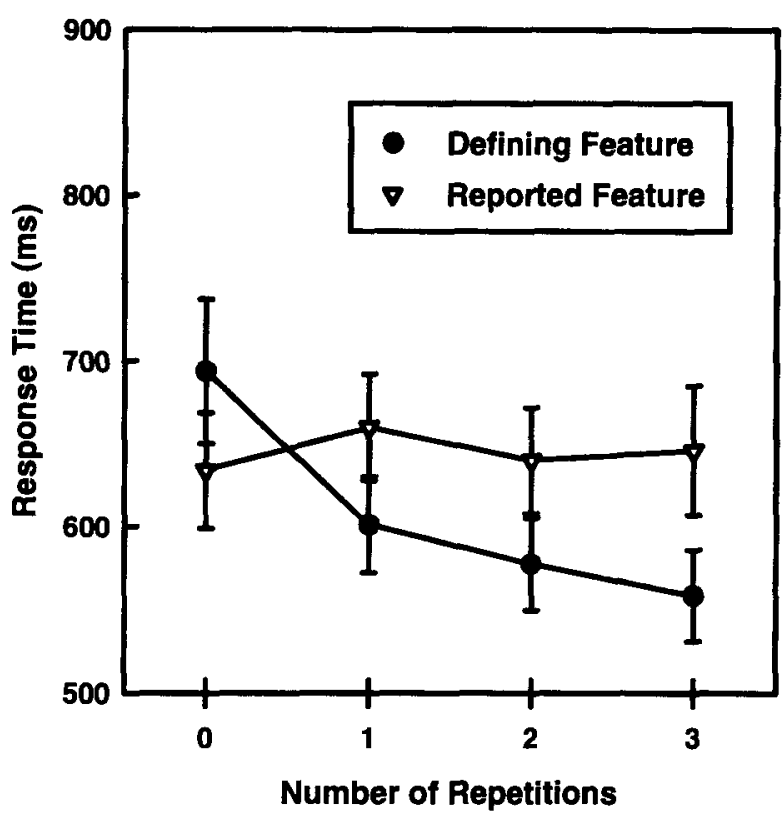

Figure 10. Experiment 3 response times as a function of the number of consecutive repetitions of the defining or the reported feature.

given that there was at least one $[F(2,22)=4.3]$. Figure 11 shows that RT was significantly influenced by the recency of the most recent trial with a matching defining feature $[F(3,33)=9.8]$. The effect of using a particular defining feature did not decay gradually. It appears that once the defining feature alternated once, a subsequent match had no effect. In fact, there was a small trend suggesting that if the most recent defining feature was not repeated, it was inhibited, and that inhibition wore off gradually.

\section{Discussion}

Experiment 3, which required the participants to adopt a top-down search strategy, showed the same robust firstorder repetition effect as that shown by Experiments 1 and 2. There was evidence of cumulative effects of multiple repetitions but no evidence of the gradual decay of a trial's influence. There was a suggestion of inhibition of higher order effects.

Even with the changes made to coerce the participants into using top-down guidance to find the targets, a strategy of letting bottom-up guidance determine target selection is possible even in this experiment. If the participants were using such a strategy, it is likely that the target would be the first or the second element attended, because two of the distractors shared a color. It seems unlikely that the participants would, on average, attend to one-and-ahalf elements (one element half the time, two elements half the time) and still respond so much faster in this experiment than in Experiments 1 and 2. Although the target colors were more salient in Experiment 3, the use of more than one distractor color should have limited the in- crease in target pop-out, at least according to Duncan and Humphreys' (1989) model of visual search. For this reason, it is likely that top-down search was used.

Thus, all models of visual search, whether they emphasize top-down or bottom-up mechanisms, must account for defining-feature repetition effects. The repetition effect seems to be a result of selection, not merely of singleton pop-out. Top-down search resulted in a different pattern of higher order defining-feature repetition effects than did bottom-up search in Experiment 1, which suggests that there may be different mechanisms underlying the repetition effect in top-down and bottom-up guidance of selection.

The pattern of higher order repetition effects in Experiment 3 was similar to that in Experiment 2, but not the same. Experiment 2 did not show cumulative effects of multiple repetitions and gave no hint of inhibition of higher order effects. It seems unlikely, therefore, that the lack of a higher order repetition effect in Experiment 2 was due primarily to the influence of top-down guidance. It is more likely that in Experiment 2, residual effects decayed too rapidly to affect more than one trial.

Although top-down guidance was involved in target selection in Experiment 3, that does not mean that bottom-up guidance was not involved. If it was involved, the repetition effects seen might be attributable to that part of target selection. Therefore, the claim that repetition effects obtain for top-down guidance to targets would be bolstered by a demonstration of repetition effects in displays in which the target was not a feature singleton. Such a demonstration would show that even when the target was not dis-

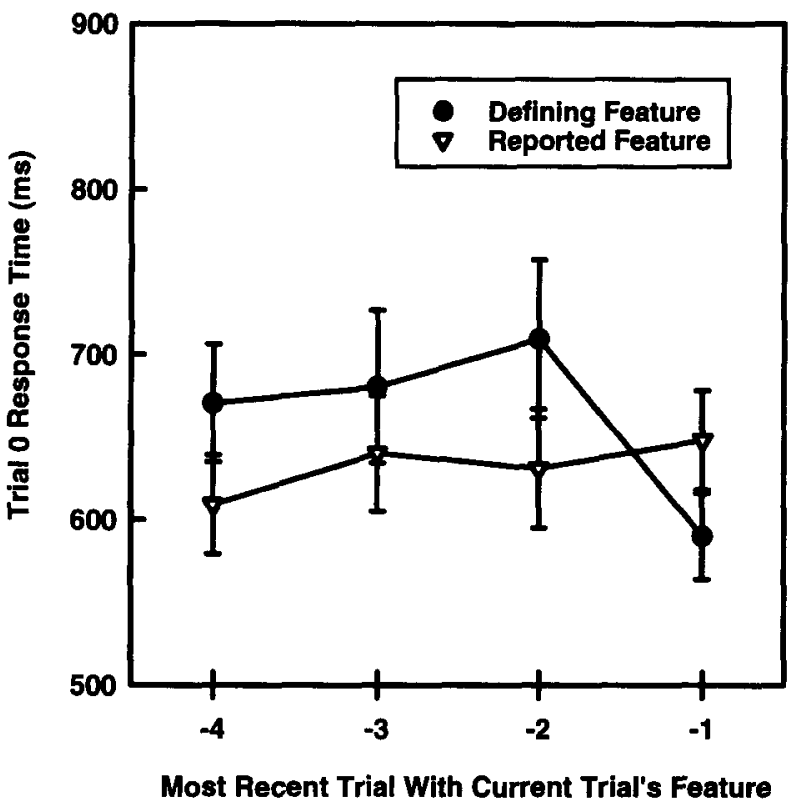

Figure 11. Experiment 3 response times as a function of the recency of the most recent trial with the same defining or reported feature. 
tinctive in terms of salience, target selection was influenced by the repetition of the defining features of the target. The aim of Experiment 4 was to demonstrate just that.

\section{EXPERIMENT 4}

In a final test of whether repetition effects are found in top-down search, in Experiment 4, the participants engaged in conjunction search. The participants searched for a solid pink or a textured purple target amid textured pink and solid purple distractors. Because neither feature that is part of the target definition is unique to the target, the salience of an individual feature cannot guide search effectively to the one target in the display. The conjunction of two features is often not salient enough to pop out of a search display (see, e.g., Treisman \& Gelade, 1980), in which case bottom-up guidance cannot contribute effectively to target selection. As such, top-down search strategies enter into conjunction search more than into singleton search or feature search. ${ }^{4}$

Display size, the number of elements in the search display, was manipulated in this experiment as well, in order to probe possible mechanisms for the repetition effect. The degree to which RT increases with each additional display element, the display size effect, reflects the priority of the target relative to the distractors. If targets are quite salient, they will have high priority no matter how many elements are in the display, and so the display size effect will be small. If, however, the target is not at all salient with respect to distractors, the display size effect will be large: The less salient the target is, the higher the likelihood that distractors will be given a priority equal to or higher than the target, and so the longer it will take to find the target. In the kind of conjunction search used in this experiment, the target should not be salient, and so the display size effect should be substantial.

Repetition of a target might affect the priority of the target, relative to distractors. For instance, if residual decaying priorities from recent trials are added to the "fresh" priorities of the current display, the target priority might be magnified. Or, as another example, the participants might tend to pick out one of the two repeated defining features and search only the subset of display elements having that feature (Bacon \& Egeth, 1997; Egeth et al., 1984; Kaptein et al., 1995; Zohary \& Hochstein, 1989). Because only half of the distractors would have the single feature focused on, this would decrease the effective display size on repeated trials and, thus, increase the priority of the target, relative to the full set of distractors. If repetition did improve the priority of the target relative to distractors, the display size effect should be smaller for repeated targets than for changed targets.

Repetition might not affect target priority, however. Changing the target definition that is used to prioritize elements might have a fixed switching cost that target repetition simply avoids. Or repetition might affect the speed of prioritizing (which is generally assumed to happen in parallel for all elements; e.g., Wolfe, 1994) without af- fecting the resulting priorities. In these cases, the display size effect should be equal for repeated and changed targets, but RT would be a fixed amount slower for changed than for repeated targets.

Pilot testing showed that switching between two conjunctively defined targets was actually quite demanding, so two manipulations were used to help ensure that the participants actually sought the intended targets. First, the random sequence was abandoned; only the alternating sequence was used, so that the participants would have maximum information before a trial about which target they were to seek. Second, a few catch trials were inserted into the experiment, in which the wrong target was presented in the display. The participants were instructed to not respond if an out-of-sequence target was presented. This manipulation allowed me to identify participants who were not trying to keep track of the sequence but merely passively searching for both targets on every trial.

\section{Method}

Participants. Twelve students ( 8 female) participated in the experiment. One reported being near-sighted even with the corrective lenses worn.

Stimuli. In every search display, five, seven, or nine rectangular bars were placed in randomly chosen positions in a circular array of 10 unmarked positions against a black background. The positions in the circular array were all centered $3.3^{\circ}$ of visual angle from the center of the circle.

The one target in the display was either solid pink or textured purple. The distractors were solid purple and textured pink. The textured bars were outlined and dotted with many small dots of the bar color. Because not all pixels were turned on, the textured stimuli were dimmer than the solid stimuli. Approximately half of the bars (two or three bars when there were five total; three or four bars when there were seven total; four or five bars when there were nine total) were randomly assigned to be vertical; the remainder were tilted $45^{\circ}$ to the left of upright.

The tone that indicated whether the target was solid pink $(1600 \mathrm{~Hz})$ or textured purple $(800 \mathrm{~Hz})$ lasted for $100 \mathrm{msec}$.

Procedure. At the start of the experiment, the participants were given instructions about the task and were told to respond as fast as possible while maintaining a high level of accuracy. The participants were given a short practice block of 20 trials of search, and then nine regular blocks of 81 trials. Feedback and rest periods were the same as those in the previous experiments.

An alternating sequence of target definitions was used in every block. Two solid pink targets were presented, followed by two textured purple targets, followed by two solid pink targets, and so forth throughout the block. Three of the trials in each block were randomly inserted catch trials. On those trials, the wrong target was presented (e.g., if the target was supposed to be solid pink, it was textured purple instead). Catch trials were included to make sure the participants were actively seeking only the target that was supposed to be presented, rather than searching for both targets on every trial. The participants were instructed at the start of the experiment to withhold making a response to catch trials.

Each trial started with the tone signaling the target for the trial. The search display appeared $750 \mathrm{msec}$ after the tone ended (a longer cue time was allowed in this experiment, because of the more complex targets), and remained on the monitor until either the participant responded or $5,000 \mathrm{msec}$ had elapsed. The next tone, starting the next trial, sounded $750 \mathrm{msec}$ after the preceding trial ended. The participants responded by pressing the left button with the left index finger if the target was tilted or the right button with the right 


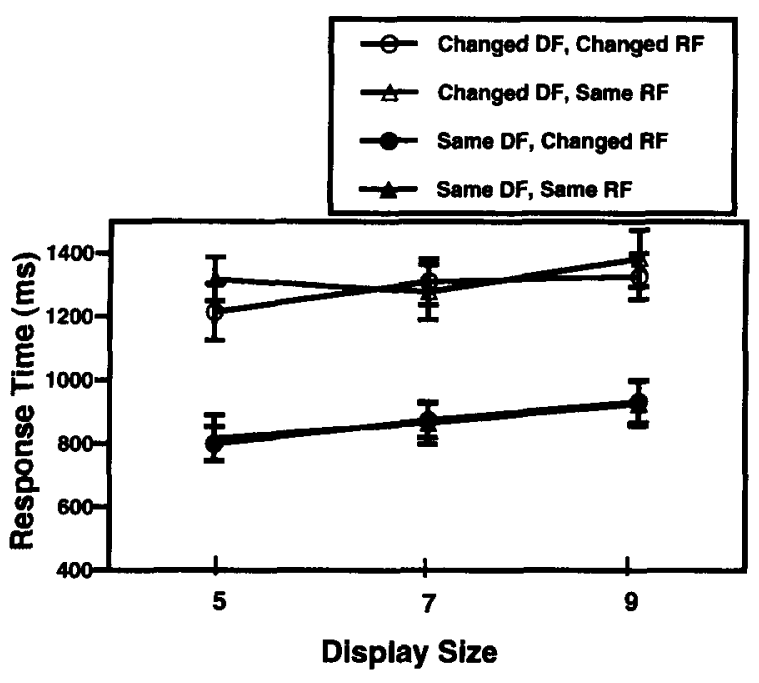

Figure 12. Experiment 4 response times as a function of definingfeatures repetition, reported-feature repetition, and display size. The participants searched for a conjunctively defined target, the definition of which was known before the trial started.

index finger if the target was vertical. Errors were handled as in all the previous experiments.

\section{Results}

The data for 2 participants were discarded because of too many responses to catch trials (the criterion for rejecting a participant's data was errors on more than $25 \%$ of the catch trials). For the remaining participants, responses were made on an average of $8 \%$ of the catch trials (ranging from $0 \%$ to $21 \%$ ). Neither the catch trials nor the trial following the catch trials was analyzed. Fewer than $2 \%$ of the trials for any participant were discarded because of particularly fast or slow responses.

First-order repetition effects. Figure 12 shows the mean RT for each of the conditions in the experiment. RTs were analyzed with a repeated measures ANOVA with three factors: defining-features repetition (same target or different target), reported-feature repetition (same or different orientation), and display size (five, seven, or nine elements).

Repeating the target definition speeded responses by $439 \mathrm{msec}[F(1,9)=169.3]$, a much larger effect than any seen in the previous experiments. Responses slowed by an average of $26.5 \mathrm{msec} / \mathrm{item}$ as display size increased $[F(2,18)=25.5]$. There was no interaction between targetdefinition repetition and display size $[F(2,18)<1]$. The insignificant trend was for the defining-features repetition effect to shrink with display size (magnitudes of the repetition effect were 458,430 , and $429 \mathrm{msec}$ at display sizes five, seven, and nine, respectively). If repetition had improved target prioritization, the repetition effect should have become larger, not smaller, as display size increased; a higher priority should have been more helpful for larger display sizes than for smaller.
Repeating the reported feature tended to slow responding, but the effect was not significant $[1,098$ and $1,076 \mathrm{msec}$ for same and different target orientations, respectively; $F(1,9)<1$ ]. There was no evidence that reported-feature repetition modulated the effect of targetdefinition repetition $[F(1,9)<1]$.

First-order effects on accuracy. Error rates averaged $2.1 \%$ (ranging from $0.2 \%$ to $4.0 \%$ across participants). Table 2 shows the number of errors made in the experiment. These error rates were analyzed with an ANOVA identical to that used to analyze response speed. Responses to repeated-target trials were more accurate than those to changed-target trials $[F(1,9)=7.9]$. The effect of target definition repetition did not appear to be due to a simple speed-accuracy tradeoff; search was both faster and more accurate when the target definition was repeated. It appears that the participants may have been slightly biased to seek repeated targets. No other effect was significant.

\section{Discussion}

In conjunction search, there was a huge effect of repeating the defining features of the target and no effect of repeating the reported feature (see, also, Treisman, 1992, for repetition effects in conjunction search) ${ }^{5}$ There was a substantial display size effect that was not affected by whether the target definition was repeated or was changed. This suggests that repetition affected the speed of prioritizing elements rather than the priorities actually assigned.

\section{GENERAL DISCUSSION}

The defining-feature repetition effect was observed not only in singleton search (Experiments 1 and 2), but also in top-down feature search (Experiment 3 ) and conjunction search (Experiment 4). The effect was not limited by the nature of the defining feature; switching the assignment of color and orientation as defining and reported features of the target changed the difficulty of search but did not eliminate or lessen the effect (Experiment 2). Multiple consecutive repetitions sometimes had cumulative effects (Experiments 1 and 3), but not always (Experiment 2). The target definitions used in preceding trials sometimes influenced current search even when other trials (and other target definitions) intervened (Experiment 1), but not always (Experiments 2 and 3), and to a lesser degree than in Maljkovic and Nakayama's (1994) experiments. Finally, repeating a conjunctively de-

Table 2

Error Rates (\%) in Experiment 4

\begin{tabular}{|c|c|c|c|c|}
\hline \multirow{2}{*}{$\begin{array}{c}\text { Display } \\
\text { Size } \\
\end{array}$} & \multicolumn{2}{|c|}{ Reported Feature Changed } & \multicolumn{2}{|c|}{ Reported Feature Repeated } \\
\hline & DF Changed & DF Repeated & DF Changed & DF Repeated \\
\hline 5 & 1.9 & 1.1 & 3.0 & 2.0 \\
\hline 7 & 3.4 & 2.8 & 2.3 & 1.8 \\
\hline 9 & 1.1 & 2.1 & 3.6 & 0.2 \\
\hline
\end{tabular}

Note-DF, defining feature. 
fined target did not affect how display size affected search time (Experiment 4).

\section{Implications for \\ Mechanisms of the Repetition Effect}

The defining-feature repetition effect did not weaken when search was guided primarily by top-down factors, rather than by target salience. Thus, top-down guidance of search is subject to repetition effects, just as bottomup guidance is. But the repetition effect in Experiment 4 was quantitatively different from the repetition effect in Experiments 1-3. It is unclear, therefore, whether the same mechanism was at work, whether a different mechanism was at work, or whether the original and a different mechanism were both at work.

The results of those experiments most clearly involving bottom-up guidance (Experiments 1 and 2) show lingering effects of repetition. After a switch occurs, there are still effects of the old definition. Thus, mechanisms that attribute repetition effects merely to the time it takes to switch to a new target definition cannot account for the repetition effects seen in singleton search (see Rogers \& Monsell's, 1995, stimulus-cued completion account of task switching for an example of such a mechanism).

In Experiment 4, repeating the target did not increase its priority. The mechanism(s) involved made prioritization more efficient but did not change the resulting priorities. This limits possible mechanisms of the repetition effect. As was stated in the introduction, it is unclear whether bottom-up guidance is obligatory in search that requires top-down guidance, such as that in Experiment 4. If bottom-up guidance was involved in Experiment 4 , the limitation to mechanisms applies to the mechanism involved in Experiments 1-3. If bottom-up guidance was not at all involved in Experiment 4, this limitation applies only to the repetition effect mechanism involved in top-down guidance.

What does this limitation mean? It means that repetition does not refine the target template or tune salience detectors to particular feature values (see, e.g., Rogers \& Monsell's, 1995, micropractice mechanism for task switching). If it did, one would expect repetition to increase the priority assigned to the target, relative to distractors. This limitation also rules out residual selection types of mechanisms, in which the facilitation or inhibition assigned to object types in one display remain in effect, to some degree, when subsequent displays appears (see, e.g., Houghton \& Tipper's, 1994, account of negative priming for an example of such a mechanism). And it rules out the possibility that both defining features are used when prioritizing changed targets, whereas only a single defining feature (perhaps the more salient one) is used when prioritizing repeated targets. If repeating the target led to the abandonment of one of the defining features, the half of the distractors that also had the abandoned feature would become low-priority distractors, and so the overall priority of the target would, on average, increase.

It should be noted that conclusions based on Experiment 4 are based on the assumption that participants do typically use both defining features to prioritize elements in conjunction search. There are those who believe that conjunction search is often accomplished by selecting a subset of elements on the basis of one defining feature and then systematically searching through that subset on the basis of the other defining feature (e.g., Kaptein et al., 1995). If our participants were doing that, the potentially changing basis of element prioritization from trial to trial renders arguments about how repetition would affect display size effects moot. For instance, if the target is prioritized on the basis of pink on one trial and on the basis of texture on the next, the priority of the target could change for reasons other than repetition effects. It would take an experiment that manipulated the proportion of each distractor type independently to address this possibility. But there are reasons to think such a manipulation may not be necessary. First, because neither of the colors was particularly salient, as compared with the other, and neither of the textures was particularly salient, as compared with the other, there was no strong bottom-up motivation to segregate the display according to color or texture. Second, the use of subset selection in conjunction search has only been demonstrated in experiments in which the proportion of the distractors with each feature varied widely from trial to trial and in which participants were usually instructed to use the strategy. Thus, it may be a strategy that is induced only with instructions or when subset selection is obviously advantageous on some trials. Third, if our participants did prioritize on the basis of single features, there is no reason to believe that the feature chosen as the basis of selection would change randomly from trial to trial.

It is quite possible that different mechanisms of repetition effects were at work in top-down and bottom-up guidance. Future research should explore this possibility. If the repetition effects in all four experiments are due to a single mechanism, however, the results are most consistent with an episodic memory mechanism for repetition effects similar to that proposed to account for negative priming (Neill, Valdes, Terry, \& Gorfein, 1992; after Logan, 1988). But this episodic memory mechanism is different from preceding ones, because it is an episodic account of prioritization alone, not of response selection. Or, if you will, the rules for prioritization are the responses that are selected. According to such an account, memory for a trial includes a representation of the features in the display and an indication of what features were prioritized higher than others. When a new display is presented, a crude preattentive map of the features in the display is created that is comparable to the representations in memory traces. That map creation triggers retrieval of traces for trials with similar display representations (displays in which similar goals were present or displays in which similar features were salient), where the prioritization rules are retrieved with the trace. If a relevant trace is retrieved before standard prioritization processing of the current display is completed, priorities are assigned to features according to the retrieved rules. Recency of trace storage speeds trace retrieval, so when the most recent 
trial is similar to the current trial in terms of attentionrelevant feature map representations, prioritization happens rapidly. If a trace cannot be retrieved rapidly because the trial is dissimilar in attention characteristics (salience, goals) from recent trials, algorithmic processing will set up prioritization rules, and prioritization time will be slower. The better that attentional characteristics are represented (e.g., when both top-down and bottom-up guidance are strongly active), the more specific and successful the retrieval will be.

Retrieval of traces might depend not only on the goals for the trial and the salience in the display, but also on spatial display configurations (see, e.g., Chun \& Jiang, 1998, for evidence that experience with specific configurations leads participants to expect targets in certain locations). However, the experiments here used displays with very limited spatial extents, so attention did not need to be moved much if at all in order to identify targets. And because display configurations did not vary systematically and did not vary much, the data from these experiments are silent about the role of display configurations on trace retrieval.

The idea that recency of trace storage affects the accessibility of traces is the crux of this episodic account. Because the displays tested were very simple, element prioritization was quite rapid, and so only very accessible traces, those still in short-term memory, had a chance to impact search. Retrieval priority, or accessibility of the traces, decays as a function of either time or interference from more recently stored traces. With more complex displays, target prioritization might benefit across longer time periods from the retrieval of episodic memories from long-term memory. For instance, Hillstrom and Logan (1998) have demonstrated long-term stimulus-specific learning of prioritization in visual search.

An episodic memory mechanism would predict that repetition would not affect target priority. If a memory trace is used to establish rules for prioritizing, the resulting rules would be the same as the retrieved rules, and so the resulting priorities need not be higher than the priorities set in the trial whose trace was retrieved. Thus, repetition affects the time to do prioritization, but not the resulting amounts of priority.

An episodic memory mechanism predicts higher order effects because short-term memory traces decay over time and retrieval of long-term memory traces are probably too slow to be faster than algorithmic processing. The more recent the matching trial, the more likely that a trace for it will still reside in short-term memory and be retrieved fast enough to be used to establish prioritization rules. The longer the string of consecutive repetitions, the greater the number of potentially useful traces available for retrieval from short-term memory. Similarity between traces (perhaps, in part, because of similarity between targets and distractors) speeds decay of retrieval priority (Posner \& Konick, 1966; Wickelgren, 1965). And if trace decay is so rapid that traces are gone from short-term memory before two trials have passed, higher order effects might not be measurable.

\section{Comparison With Other Repetition Effects}

A number of repetition phenomena are under investigation currently, and the defining-feature repetition effect is similar, to a greater or lesser degree, to some of them. In particular, it seems useful to compare the definingfeature repetition effect with task switching, the reportedfeature repetition effect, and negative priming.

Task switching. Switching from one task to another often exacts an RT cost (Jersild, 1927; Spector \& Biederman, 1976). For example, if participants see a serially presented list of numbers and must either repeat the same arithmetic operation for every number (e.g., add 3 to each number or subtract 3 from each number) or alternate between operations when processing the list (add 3 to the first number, subtract 3 from the next, and so on), RTs will be slower for the alternating-task condition than for the repeating-task condition. Even when participants are told exactly what task to switch to and are given a long time to make the switch, the first trial of the new task shows a switch cost (Allport, Styles, \& Hsieh, 1994; Rogers \& Monsell, 1995). Thus, task switching, rather than being completely volitional, appears to require triggering by stimuli. And the alternation disadvantage often disappears when the stimuli themselves cue which task to perform (Allport et al., 1994; Jersild, 1927; Spector \& Biederman, 1976). For these reasons, the cost of switching tasks is thought to reflect involuntary interference between two or more potentially relevant task sets.

Although discussion up to now has described the defining-feature repetition effect as a benefit of repeating an attentional set, it can be thought of as a cost of switching attentional set. This frames it for comparison with task switching. Switching attentional sets appears to work differently than switching tasks. Although both have voluntary and involuntary effects, task switching appears to affect only the first trial in the new task (Rogers \& Monsell, 1995), but switching attentional set shows effects on multiple trials, at least some of the time (Experiment 1; Maljkovic \& Nakayama, 1994).

Reported-feature repetition. In standard choice RT paradigms, a response repeated from the preceding trial is more rapid than a response that is different, as long as RSI is brief (Bertelson, 1961). When RSI is long or when alternations are more probable than repetitions, expectancy sometimes results in faster responses for alternation trials than for repetition trials (see, e.g., Bertelson, 1961; Bertelson \& Renkin, 1966; Hale, 1967; Soetens et al., 1985; Soetens et al., 1984; Umiltà et al., 1972). But in experiments that carefully equate the probability of response repetition and alternation, a repeated response is often made more rapidly than a changed response (Bertelson, 1961; Hyman, 1953; Kornblum, 1969; Soetens et al., 1985). The repetition effect is influenced by both the stimulus and the response (Bertelson, 1965; Rabbitt, 
1968). A more important determinant of the effect is stimulus-response compatibility (Bertelson, 1963; Keele, 1969; Pashler \& Baylis, 1991; Soetens et al., 1985), and so current theories attribute the response repetition effect to the stimulus-response mapping stage of processing (e.g., Pashler \& Baylis, 1991).

The defining-feature repetition effect seems to be similar but not identical to the reported-feature repetition effects. Both are affected by expectancy for repetition: the greater the foreknowledge of what stimulus to expect, the larger the repetition effect (Experiments 1 and 2; Bertelson, 1961). But the temporal characteristics of the phenomena are different. In reported-feature repetition effects, expectation plays only a minor role at short RSIs $(50-500 \mathrm{msec})$ but plays a more important role than automaticity at RSIs upward of $1,000 \mathrm{msec}$ (Kornblum, 1973; Soetens et al., 1985). In contrast, the component of the defining-feature repetition effect that is not influenced by expectancy is still strong at RSIs of approximately $900 \mathrm{msec}$ (Experiments 1 and 2) or even 2,500 msec (Maljkovic \& Nakayama, 1994). And perhaps the largest difference seen in these experiments is that repeating the reported feature often slowed responses, whereas repeating the defining feature speeded responses in all the experiments.

Negative priming. There are also striking similarities between the defining-feature repetition effect and negative priming, a special form of a reported-feature repetition effect. Negative priming is a phenomenon typically studied by having participants view one target and one distractor on each trial and report a feature (usually the identity) of the target. Responses are fast if the target identity is repeated from the preceding trial, and responses are slow if the target identity matches the preceding trial's distractor identity (Tipper, 1985), particularly if the stimuli in the experiment are used repeatedly as both target and distractors (Malley \& Strayer, 1995). The slowing of responses when the preceding trial's distractor identity matches the current trial's target identity has been labeled negative priming. For both negative priming and the defining-feature repetition effect, a preceding trial can influence the current trial even when another trial intervenes (Experiment 1; Tipper, Weaver, Cameron, Brehaut, \& Bastedo, 1991). Episodic memory mechanisms are strong contenders in accounting for each (Neill, 1997; Neill et al., 1992; Treisman, 1992; but see Moore, 1994; Strayer, 1995). ${ }^{6}$

Because negative priming experiments typically manipulate only the reported features of display items, it is unclear whether the defining-feature repetition effect is related. Because there are seldom more than two display items, the target and one distractor, it seems possible that target selection and response selection are less clearly delineated parts of the task in negative priming experiments. But of the two projects that I know varied the defining feature of the target and looked for negative priming in the defining rather than the reported feature, both found negative priming in some but not all experiments (Hillstrom, 1995; Tipper, Weaver, \& Houghton, 1994).

The episodic model of the defining-feature repetition effect was greatly influenced by the Neill et al. (1992) episodic model of negative priming. A more recent model that is also, to some degree, episodic attributes negative priming to an automatic mechanism that searches for whether a display is identical to the one that was just responded to, in order to determine whether the response to the display is already known (Milliken, Joordens, Merikle, \& Seiffert, 1998). The temporal discrimination model may be a reasonable model of reported-feature repetition effects, but in its current state of development, it cannot account for the different effects of the defining feature and the reported feature.

\section{CONCLUSIONS}

The experiments reported here explored the definingfeature repetition effect in visual search. They showed that the effect occurs not only in bottom-up guidance of search, but also in top-down guidance of search. The experiments showed that the higher order repetition effects observed in previous research are not as robust as firstorder effects. An episodic memory mechanism provides the most satisfactory account of repetition effects.

\section{REFERENCES}

Allport, D. A., Styles, E. A., \& Hsieh, S. (1994). Shifting intentional set: Exploring the dynamic control of tasks. In C. Umiltà \& M. Moscovitch (Eds.), Attention and performance XV: Conscious and nonconscious information processing (pp. 421-452). Cambridge, MA: MIT Press, Bradford Books.

BACON, W. F., \& EGETH, H. E. (1994). Overriding stimulus-driven attentional capture. Perception \& Psychophysics, 55, 485-496.

BACON, W. F., \& EGETH, H. E. (1997). Goal-directed guidance of attention: Evidence from conjunctive visual search. Journal of Experimental Psychology: Human Perception \& Performance, 23, 948-961.

BerTElson, P. (1961). Sequential redundancy and speed in the serial two-choice responding task. Quarterly Journal of Experimental Psychology, 13, 90-102.

BERTELSON, P. (1963). S-R relationships and reaction time to new versus repeated signals in a serial task. Journal of Experimental Psychology, 65, 478-484.

BERTELSON, P. (1965). Serial choice reaction time as a function of response versus signal repetition. Nature, 206, 217-218.

Bertelson, P., \& Renkin, A. (1966). Reaction times to new versus repeated signals in a serial task as a function of response-signal time interval. Acta Psychologica, 25, 132-136.

Chun, M. M., \& JiAng, Y. (1998). Contextual cueing: Implicit learning and memory of visual context guides spatial attention. Cognitive Psychology, 36, 28-71.

Duncan, J. (1985). Visual search and visual attention. In M. I. Posner \& O. S. M. Martin (Eds.), Attention and performance XI (pp. 85106). Hillsdale, NJ: Erlbaum.

Duncan, J., \& Humphreys, G. W. (1989). Visual search and stimulus similarity. Psychological Review, 96, 433-458.

EGETH, H. E. (1977). Attention and preattention. In G. H. Bower (Ed.), The psychology of learning and motivation: Advances in theory and research (Vol. 11, pp. 277-320). New York: Academic Press. 
Egeth, H. E., Virzi, R. A., \& Garbart, H. (1984). Searching for conjunctively defined targets. Journal of Experimental Psychology: Human Perception \& Performance, 10, 32-39.

FLetCHER, B. C. (1981). Wholistic and analytic stimulus processing: The development of selective perceptual strategies. Quarterly Journal of Experimental Psychology, 33A, 167-183.

Folk, C. L., Remington, R. W., \& Johnston J. C. (1992). Involuntary covert orienting is contingent on attentional control settings. Journal of Experimental Psychology: Human Perception \& Performance, 18, 1030-1044.

Gibson, B. S., \& KelSEY, E. M. (1998). Stimulus-driven attentional capture is contingent on attentional set for display-wide visual features. Journal of Experimental Psychology: Human Perception \& Performance, 24, 699-706.

HALE, D. J. (1967). Sequential effects in a two-choice serial reaction task. Quarterly Journal of Experimental Psychology, 19, 133-141.

HiLlstrom, A. P. (1995, November). Singleton pop-out: Facilitation of uniqueness or inhibition of similarity? Paper presented at the $36 \mathrm{th}$ Annual Meeting of the Psychonomic Society, Los Angeles.

Hillstrom, A. P., \& Logan, G. D. (1998). Decomposing visual search: Evidence of multiple item-specific skills. Journal of Experimental Psychology: Human Perception \& Performance, 24, 1385-1398.

Hillstrom, A. P., \& YANTIS, S. (1994). Visual motion and attentional capture. Perception \& Psychophysics, 55, 399-411.

Hommel, B. (1998). Event files: Evidence for automatic integration of stimulus-response episodes. Visual Cognition, 5, 183-216.

Houghton, G., \& TipPER, S. P. (1994). A model of inhibitory mechanisms in selective attention. In D. Dagenbach \& T. H. Carr (Eds.), Inhibitory mechanisms in attention, memory, and language (pp. 53-112). San Diego: Academic Press.

HymaN, R. (1953). Stimulus information as a determinant of reaction time. Journal of Experimental Psychology, 45, 188-196.

JersiLd, A. T. (1927). Mental set and shift. Archives of Psychology, 14 (Whole No. 89).

Kaptein, N. A., Theeuwes, J., \& van der Heijden, A. H. C. (1995). Search for a conjunctively defined target can be selectively limited to a color-defined subset of elements. Journal of Experimental Psychology: Human Perception \& Performance, 21, 1053-1069.

KeELE, S. W. (1969). Repetition effect: A memory-dependent process. Journal of Experimental Psychology, 80, 243-248.

KoRnBLUM, S. (1969). Sequential determinants of information processing in serial and discrete choice reaction time. Psychological Review, 76, 113-131.

Kornblum, S. (1973). Sequential effects in choice reaction time: A tutorial review. In S. Kornblum (Ed.), Attention and performance IV (pp. 259-288). New York: Academic Press.

Lockhead, G. R., Gruenewald, P., \& King, M. (1978). Holistic vs. attribute repetition effects in classifying stimuli. Memory \& Cognition, 6, 438-445.

LOGAN, G. D. (1988). Toward an instance theory of automatization. Psychological Review, 95, 492-527.

Maljkovic, V., \& Nakayama, K. (1994). Priming of pop-out: I. Role of features. Memory \& Cognition, 22, 657-672.

Malley, G. B., \& Strayer, D. L. (1995). Effect of stimulus repetition on positive and negative identity priming. Perception \& Psychophysics, 57, 657-667.

MARR, D. (1982). Vision. New York: Freeman.

Milliken, B., Joordens, S., Merikle, P. M., \& Seiffert, A. E. (1998). Selective attention: A reevaluation of the implications of negative priming. Psychological Review, 105, 203-229.

MOORE, C. M. (1994). Negative priming depends on probe-trial conflict: Where has all the inhibition gone? Perception \& Psychophysics, 56, 133-147.

NEILL, W. T. (1997). Episodic retrieval in negative priming and repetition priming. Journal of Experimental Psychology: Learning, Memory, \& Cognition, 23, 1291-1305.

Neill, W. T., Valdes, L. A., Terry, K. M., \& Gorfein, D. S. (1992). Persistence of negative priming: II. Evidence for episodic trace retrieval. Journal of Experimental Psychology: Learning, Memory, \& Cognition, 18, 993-1000.
Pashler, H., \& Baylis, G. (1991). Procedural learning: 2. Intertrial repetition effects in speeded-choice tasks. Journal of Experimental Psychology: Learning. Memory, \& Cognition, 17, 33-48.

Posner, M. I., \& KonICK, A. F. (1966). On the role of interference in short-term retention. Journal of Experimental Psychology, 72, 221231.

RabBitT, P. M. A. (1968). Repetition effects and signal classification strategies in serial choice-response tasks. Quarterly Journal of Experimental Psychology, 20, 232-240.

Rabitt, P. M. A., Cumming, G. D., \& Vyas, S. M. (1977). An analysis of visual search: Entropy and sequential effects. In S. Dornic (Ed.), Attention and performance VI (pp. 363-368). Hillsdale, NJ: Erlbaum.

Rabitt, P. [M. A.], Cumming, G. [D.], \& Vyas, S. [M.] (1979). Modulation of selective attention by sequential effects in visual search tasks. Quarterly Journal of Experimental Psychology, 31, 305-317.

Rogers, R. D., \& Monsell, S. (1995). Costs of a predictable switch between simple cognitive tasks. Journal of Experimental Psychology: General, 124, 207-231.

Soetens, E., Boer, L. C., \& Hueting, J. E. (1985). Expectancy or automatic facilitation? Separating sequential effects in two-choice reaction time. Journal of Experimental Psychology: Human Perception \& Performance, 11, 598-616.

Soetens, E., Deboeck, M., \& Hueting, J. (1984). Automatic aftereffects in two-choice reaction time: A mathematical representation of some concepts. Journal of Experimental Psychology: Human Perception \& Performance, 10, 581-598.

Spector, A., \& Biederman, I. (1976). Mental set and mental shift revisited. American Journal of Psychology, 89, 669-679.

STRAYER, D. L. (1995, November). Negative priming and stimulus repetition. Paper presented at the 36th Annual Meeting of the Psychonomic Society, Los Angeles.

Theeuwes, J. (1991). Cross-dimensional perceptual selectivity. Perception \& Psychophysics, 50, 184-193.

TIPPER, S. P. (1985). The negative priming effect: Inhibitory priming by ignored objects. Quarterly Journal of Experimental Psychology, 37, 571-590.

Tipper, S. P., Weaver, B., Cameron, S., Brehaut, J. C., \& Bastedo, J. (1991). Inhibitory mechanisms of attention in identification and localization tasks: Time-course and disruption. Journal of Experimental Psychology: Learning, Memory, \& Cognition, 17, 681-692.

TipPER, S. P., Weaver, B., \& Houghton, G. (1994). Behavioral goals determine inhibitory mechanisms of selective attention. Quarterly Journal of Experimental Psychology, 47A, 809-840.

Treisman, A. (1992). Perceiving and re-perceiving objects. American Psychologist, 47, 862-875.

Treisman, A., \& Gelade, G. (1980). A feature-integration theory of attention. Cognitive Psychology, 12, 97-136.

Umiltì, C., SNyder, C., \& SNyder, M. (1972). Repetition effect as a function of event uncertainty, response-stimulus interval, and rank order of the event. Journal of Experimental Psychology, 93, 320-326.

WiCKELGREN, W. (1965). Acoustic similarity and retroactive interference in short-term memory. Journal of Verbal Learning \& Verbal Behavior, 4, 53-61.

WoLFE, J. M. (1994). Guided Search 2.0: A revised model of visual search. Psychonomic Bulletin \& Review, 1, 202-238.

YANTIS, S., \& EgETh, H. E. (1999). On the distinction between visual salience and stimulus-driven attentional capture. Journal of Experimental Psychology: Human Perception \& Performance, 25, 661-676.

ZOHARY, E., \& HoCHSTEIN, S. (1989). How serial is serial processing in vision? Perception, 18, 191-200.

\section{NOTES}

1. The RSIs used in these experiments $(800-1,500 \mathrm{msec})$ were shorter than those used in Maljkovic and Nakayama's (1994) experiments $(2,500 \mathrm{msec})$. This change was made mostly just to keep the pace of the experiment fast enough to keep the participants engaged in the task. If visual search repetition effects are like choice RT repetition effects, the effect of this change was probably to strengthen the repetition 
effect, particularly the more automatic part of it (see, e.g., Bertelson, 1961; Bertelson \& Renkin, 1966; Hale, 1967; Soetens, Boer, \& Hueting, 1985; Soetens, Deboeck, \& Hueting, 1984; Umiltà, Snyder, \& Snyder, 1972)

2. Only the data in the blocks with random trial sequences were analyzed for higher order repetition effects, because the alternating sequence had artificial constraints on which trials and how many consecutive trials had the same defining feature as the current trial.

3. RT could also have been slowed for other reasons, such as different response-selection mappings in the two experiments. Because neither mapping (color to finger and orientation to finger) appears to be particularly congruent or particularly incongruent, this influence of the different response-selection mappings is assumed to be negligible.

4. Note that salience could, and probably does, play a role in guidance of attention in this search task. For instance, salience may contribute to a choice of a subset of elements to search first on the basis of one of the two target-defining features (Egeth, Virzi, \& Garbart, 1984; Kaptein,
Theeuwes, \& van der Heijden, 1995). All that is claimed is that topdown guidance plays a much stronger role in conjunction searches like this one than in feature search or singleton search, because the target is not more salient than the majority of the distractors.

5. Targets in the research reported there were likely to appear in certain locations. They found a repetition effect only when the target appeared in the expected location. Target-location contingency could well have made target location a defining feature, albeit an imperfect one.

6. Negative priming could, in principle, coexist with defining-feature repetition effects in visual search experiments like as those designed by Maljkovic and Nakayama (1994). However, visual search displays typically include multiple distractors that have various response features, so the influence of one distractor's response-related feature is likely to cancel out the influence of another distractor's response-related feature.

(Manuscript received September 16, 1996; revision accepted for publication April 27, 1999.) 